\title{
Temperature-dependent crossover in fractional quantum Hall edges in the presence of Coulomb interaction
}

\author{
Wenjun Zheng and Yue Yu \\ Institute of Theoretical Physics, Academia Sinica, Beijing 100080, China
}

()

Based on a newly derived microscopic fractional quantum Hall edge model, we study its thermodynamics at finite temperature. For the dressed energy spectrum a critical energy scale determined by the temperature exists, below which the refractive dispersion which is essential to the model's bosonization to a Luttinger liquid is smeared. According to this observation, a temperature-dependent crossover picture is proposed, and applied to the analysis of a recent tunneling experiment in comparison with the standard Luttinger liquid theory, and a better fit to the features of the measured conductance-temperature curve is achieved. We also consider the role of the Coulomb interaction in the thermodynamics and find that a crossover exists during which the influence of the Coulomb interaction is suppressed with the increase of temperature.

PACS numbers: 73. 40. Hm, 71. 10. +x, 71. 27. +a

Since the sensational discovery of fractional quantum Hall effect(FQHE) in 1982 [1], many efforts have been devoted to the understanding of this fascinating $2 \mathrm{D}$ strongly correlated system [2]. In the theoretical aspect, progress has been made to account for its low energy physics which is determined by the FQHE edge states because of the gapful bulk excitations. Compared with the integer quantum Hall edge states which are well understood in a chiral Fermi liquid picture [3], the description of the FQHE edge is much more subtle. There is still some controversy in the real effects of electron correlations. One of the most concerned questions is whether the fractional edge is a Fermi liquid (FL)or a Luttinger liquid(LL) 顿. From the viewpoint of the macroscopic effective theory, Wen advanced the chiral Luttinger liquid (CLL) picture [0], which emphasizes the non-Fermi liquid nature of the FQHE edge states due to strong correlations. Some predictions [6] [7] based on CLL have been corroborated in a recent tunneling experiment between $\nu=1 / 3$ fractional edges [8]. Meanwhile, a microscopic composite fermion (CF) [9] picture was used to construct a mean field level edge model, which is basically a Fermi liquid one [10]. It is not surprising that this naive mean field theory also found experimental supports from the measurement of the anti-dot $\mathrm{AB}$ effect [11] and the resonant tunneling width [12], for its bulk counterpart has already achieved impressive experimental successes. In a recent study [13] 14, we succeed in relating the above two pictures with each other by giving a microscopic derivation of the CLL model 15]. In our derivation, we perform a composite fermion transformation on the electron Hamiltonian, which attaches an even number of flux quanta onto the electrons to form the composite particles. While the statistical gauge field interactions between the edge particles and the bulk ones are treated at a mean field level, the gauge interactions between the edge particles are taken care of explicitly, which lead to the square inverse interaction characteristic of the Calogero Sutherland model(CSM) [16]. In this way, we can show that the FQHE edge states are described by the CSM with the residue interactions between the CFs treated as perturbations. Then by means of bosonization and consideration of the chirality, we finally arrive at Wen's CLL theory. The role of the residue short-range interactions is also considered and proven to be unable to change the critical exponents of the CSM because of the chirality [17]. In this letter, we are going to reconcile the conflicts between the Fermi liquid and the Luttinger liquid descriptions by studying the thermodynamics of the present microscopic edge model at finite temperature. We argue that the refractive dispersion of the dressed energy which is essential to the CSM's bosonization to a LL is smeared in the "window" opening around the Fermi point, whose width is set by the temperature. The physical processes involving only excitations of much lower energy scales will show FL like behaviors, while those including higher energy excitations will undertake a crossover to LL with the increase of energy scales. This mechanism $[18$ makes it possible to reconcile between the experimentally detected LL behaviors and the FL ones. We then apply this intuitive crossover picture to the analysis of a recent tunneling experiment from a normal metal to the $\nu=1 / 3$ fractional edge, and succeed in explaining two features of the $G-T$ data which are inconsistent with the standard LL prediction. We also discuss the role of the Coulomb interaction in the thermodynamics, and argue that finite temperatures tend to suppress its effects.

Before starting with the discussion of the thermodynamics, we first give a concise review of the microscopic edge model. Both the thermodynamics and the bosonization of it stem from the well-known thermodynamic Bethe ansatz (TBA)equation [19] that reads

$$
\epsilon(k)=\epsilon_{0}(k)+\frac{T}{2 \pi} \int_{-\infty}^{\infty} \Phi^{\prime}\left(k-k^{\prime}\right) \ln \left(1+e^{-\frac{\epsilon\left(k^{\prime}\right)}{T}}\right) d k^{\prime}
$$

where $\epsilon(k)$ is the dressed energy, $\epsilon_{0}(k)$ stands for the bare energy given by $\epsilon_{0}(k)=k^{2}-\mu, \mu$ is the chemical 
potential , $\Phi(k)$ represents the phase shift function of the model interaction, which in the CSM reads $\Phi_{C S}(k)=$ $(\lambda-1) \pi \operatorname{sgn}(k)$, and the model interaction is $V_{C S}(x)=$ $\frac{\lambda(\lambda-1)}{4}\left[\sin \left(\frac{x}{2}\right)\right]^{-2}$. The free energy at temperature $T$ is given by the dressed energy as follows,

$$
\begin{aligned}
F & =U-T S \\
& =-T \int_{-\infty}^{\infty} \rho_{0} \ln \left(1+e^{-\frac{\epsilon(k)}{T}}\right) d k
\end{aligned}
$$

where $\rho_{0}=\frac{1}{2 \pi}$. In order to bosonize the low energy effective theory of the CSM, one goes to the zero temperature limit [20], and the TBA equation reduces to

$$
\epsilon(k)=\epsilon_{0}(k)-\frac{1}{2 \pi} \int_{-k_{F}}^{k_{F}} \Phi^{\prime}\left(k-k^{\prime}\right) \epsilon\left(k^{\prime}\right)
$$

and one obtains the following refractive dispersion of the dressed energy

$$
\epsilon_{ \pm}(k)= \begin{cases} \pm v_{F}\left(k \mp k_{F}\right)+k_{F}^{2}, & |k|>k_{F} \\ \pm v_{F}\left(k \mp k_{F}\right) / \lambda+k_{F}^{2}, & |k|<k_{F}\end{cases}
$$

where $v_{F}, k_{F}$ are the Fermi velocity and the Fermi momentum respectively. Based on this dispersion relation and the ground state energy formula $E=\int_{-k_{F}}^{k_{F}} \rho_{0} \epsilon(k) d k$, one can bosonize the CSM and arrive at the low energy fixed point of LL, with the characteristic exponent $g=\lambda$. We emphasize the importance of the refractive dispersion, which is essential to the conclusion of non-Fermi $\operatorname{liquid}(g \neq 1)$ in the CLL picture.

Then we turn to the situation of finite temperature, and calculate the dispersion of the dressed energy near the Fermi point. A momentum scale $k_{T}$ set by $T$ is given by $k_{T}=T / v_{F}$. For $\left|k-k_{F}\right|<<k_{T}$, or $|\epsilon(k)|<<T$, the TBA equation reduces to

$$
\epsilon(k)=\epsilon_{0}(k)+T(\lambda-1) \ln \left(1+e^{-\frac{\epsilon(k)}{T}}\right)
$$

One can expand it to the $O\left(\frac{\epsilon(k)}{T}\right)$ order, and obtain

$$
\left.\epsilon(k)=\frac{2}{1+\lambda} \epsilon_{0}(k)+\text { const }+O\left[\left(\frac{\epsilon(k)}{T}\right)^{2}\right)\right]
$$

where the temperature-dependent part of $\epsilon(k)$ is absorbed into the chemical potential to satisfy the condition $\epsilon\left(k_{F}\right)=0$, with the remaining temperature-independent term $\frac{2}{1+\lambda} \epsilon_{0}(k)$ still meaningful as a physical energy. One can see that the refractive dispersion at $T=0$ is smeared near the Fermi point at finite temperature. Then we consider the situation of $\left|k-k_{F}\right|>>k_{T}$, or $|\epsilon(k)|>>T$. One can easily obtain the dispersion relation similar to that of zero temperature as follows

$$
\epsilon_{ \pm}(k)= \begin{cases}\epsilon_{0}(k)+O\left(e^{\frac{-|\epsilon(k)|}{T}}\right), & |k|>k_{F}, \\ \epsilon_{0}(k) / \lambda+O\left(e^{\frac{-|\epsilon(k)|}{T}}\right), & |k|<k_{F} .\end{cases}
$$

where the refractive dispersion is restored. Therefore the dispersion relation of the dressed energy that is essential to the CSM's bosonization to a LL is smeared at energy scales lower than $T$, where the apparent "refractive index" turns from $\lambda$ to 1 , and a Fermi liquid like low energy effective theory is obtained. In this way both FL and LL behaviors can be accommodated at finite $T$, depending on the energy scales involved in different physical processes.

Let us first discuss the equilibrium thermodynamics, which is determined by the free energy $F$ in the canonical ensemble formulation, and $F$ can be divided into the sum of two parts as follows

$$
\begin{aligned}
F=F_{l}+F_{h} & \\
F_{l} & =-T \sum_{r=-1,1_{\left|k-r k_{F}\right|<k_{T}}} \int_{0} \rho_{0} \ln \left(1+e^{-\frac{\epsilon(k)}{T}}\right) \\
F_{h} & =-T \sum_{r=-1,1_{\left|k-r k_{F}\right|>k_{T}}} \rho_{0} \ln \left(1+e^{-\frac{\epsilon(k)}{T}}\right)
\end{aligned}
$$

and $F_{l}$ is more important because low energy excitations contribute more to the temperature-dependent part of $F$ which determines the thermodynamics. One can see that $F_{l}$ contains the contribution from the FL like dressed energy spectrum at low energy scales, while $F_{h}$ contains the contribution from the LL related refractive spectrum at large energy scales. So the thermodynamic functions derived from $F$ have mixed properties of $\mathrm{FL}$ and LL. Attempts to clarify the FL/LL ambiguity, or to extract the corresponding characteristic parameters (such as $v_{F}, v_{N}$ of LL) by measuring the equilibrium thermodynamic quantities are expected to be improper. However, experiments designed to probe predominantly low energy excitations or high energy ones (relative to $T$ ) should be able to discriminate between FL and LL behaviors, such as the measurement of the temperature and the bias voltage dependences of the tunneling conductance $G(V, T)$. We first consider the situation of tunneling from a normal metal to a $1 / 3$ fractional edge at fixed $T$. Since $e V$ corresponds to the energy scale of the most relevant excitations probed by the measurement of $G(T, V)$, one can expect the behavior of $G(T, V)$ to undertake a crossover from FL to LL with the increase of $V$. For $e V<<T$, the $V$ independent behavior is expected, which results from the FL like low energy excitations; while for $\mathrm{eV}>>T$ the power law property of LL is restored, that is

$$
G(T, V) \propto \begin{cases}V^{2}, & e V>>T \\ V^{0}, & e V<<T\end{cases}
$$

To verify the above qualitative prediction, we cite the experimental results of Chang et al. 22 for comparison. 
In Fig.1a, the measured $I-V$ relation at fixed $T$ experiences a crossover from $I \propto V$ to $I \propto V^{3}$ near $e V \approx T$, which means a corresponding crossover for $G(T, V)$ from $V^{0}$ to $V^{2}$, as predicted above. The quantitative result of the $I-V$ curve based on the crossover picture is shown in Fig.1b for comparison. One can see that the fit is satisfactory. However, a good fit to the experiments can also be achieved by means of the scaling relation derived from the standard LL theory. In order to demonstrate that the current crossover picture is beyond an equivalent of the LL theory, we will turn to the situation of variable $T$, where the present picture is able to describe more features of the measured $G-T$ data than the standard LL theory.

To make a quantitative comparison between the prediction of the crossover picture and that of the LL theory, we first cite the basic results of the latter. By taking both $T$ and $V$ dependences into account, Kane and Fisher suggest a universal scaling form for the $I-V$ relation that holds in the limit $G_{\text {tun }}<<G_{\text {Hall }}=e^{2} / 3 h$ (suppose $\nu=1 / 3$ ). For tunneling into the $1 / 3$ edge from a normal metal, it is given by [21]

$$
I \propto T^{\alpha}\left[x+x^{\alpha}\right]
$$

where $x=\frac{e V}{2 \pi T}, \alpha=3$. Therefore $G(T, V)$ can be written as

$$
G(T, V) \propto T^{2}+B V^{2}
$$

where $B$ is a constant. We then cite the experimental results of reference [22] in Fig.2a, which will be the bases of the following discussions and comparisons. After a careful examination of Fig.2a, one can discover two features that are inconsistent with eq(10), but can be understood qualitatively within the crossover picture. First, The two curves of $G(T, V)$ tend to saturate with the increase of $T$, before the one with lower $V$ warps up (the so called meander behavior in ref 22]), while the other with higher $V$ continues to saturate when approaching the gapful hole excitations. That means the exponent of $T$ tends to decrease with the increase of $T$. However, according to eq(10), the exponent should go up to 2 with the $V^{2}$ term being suppressed by larger $T^{2}$. This feature can be understood in the crossover picture. When we increase $T$ while fix $V$, more and more relevant excitations belong to the FL like part of the spectrum, which has the effect of gradually lowering the exponent from the LL value 2 to the FL value 0, although the crossover is disrupted by the gapful excitations. Secondly, we note that the $G(T, V)$ curve with larger $V$ has larger exponent 23. But eq(10) predicts the opposite result, since larger $V^{2}$ weakens the power law behavior of $T^{2}$. This again can be explained qualitatively here, because larger $V$ means to probe higher energy excitations which tend to reach the LL region of the dressed energy spectrum, and as a result leads to larger exponent.

In order to give a quantitative justification of the above argument, we calculate $G(T, V)$ by using the following tunneling density of states

$$
N(E) \propto \begin{cases}N_{0}, & E<<T, \\ E^{2}, & E>>T .\end{cases}
$$

$N(E)$, for $E$ near the order of $T$, corresponds to a crossover between the above two limiting regions [24. $N_{0}$ does not depend on $T$ because $\frac{2}{1+\lambda} \epsilon_{0}(k)$ in eq(5) is $T$-independent . $G(T, V)$ is given by

$G(T, V) \propto \int_{-\infty}^{\infty}\left[f^{\prime}(E+e V)+f^{\prime}(E-e V)\right](1-f(E)) N(E) d E$

where $f(E)=1 /\left(1+e^{E / T}\right)$ is the Fermi distribution function. The numerical results are shown in Fig2.b. The basic features of Fig.2a are reproduced satisfactorily. Even the warping up behavior is manifest, which can be analyzed as follows. According to the expression of $N(E)$, the contributions of the LL part and the FL like part can be separated in $G(T, V)$ as

$$
G(T, V)=W_{F L}(V / T)+W_{L L}(V / T) T^{2}
$$

where $W_{F L}(x)$ and $W_{L L}(x)$ are weight functions of the two contributions. With the increase of $T$ from the order of $V$, the variations of weight functions at first dominate the $G-T$ behavior by transferring weight from $W_{L L}$ to $W_{F L}$, which leads to the slight saturation of $G(T, V)$. However, if we further increase $T$, the weight functions themselves tend to saturate, while the $T^{2}$ term begins to dominate the $G-T$ behavior, which results in the warping up tendency. With the further increase of $T$, the exponent rapidly approaches the LL value 2 . For large enouch $V$, the warping up behavior may be suppressed by the approaching of the gapful excitations which tend to saturate $G_{\text {tun }}$ to $G_{\text {Hall }}=e^{2} / 3 h$. This explains the loss of meander in the experimental curve with larger $V$. Therefore, the FL behavior of $G(T, V)$ only manifests itself in a small interval of $T$ on the condition that $V$ is small enough, although the contribution of the FL like excitations persists at the high temperature limit. The detailed fit between theory and experiments will be reported elsewhere 26.

From the above discussions and comparisons, one can see that a smooth crossover from LL to FL like behaviors occurs with the increase of $T$. If we take into account the finite size effects, the crossover temperature $T_{c}$ is roughly determined by the length scale $L$ as $T_{c}=v_{F} \frac{h}{L}$. For $T<T_{c}$, the FL window near the Fermi point is actually absent, while for $T>T_{c}$, the FL like effects begin to show up. In this way, both the finite temperature effects and the finite size effects are coherently related, and they are complementary to each other in that whenever one becomes weaker the other will turn stronger.In typical experiments on the FQHE edge tunnelings in a mesoscopic sample, $T_{c}$ can be as large as the order of $T$, and one can only measure a mixed property of FL and LL. For instance, in ref [22] where the tunneling into the $\nu=1 / 3$ fractional edge is probed, the measured exponents $(\alpha-1=1.75,1.5)$ in the $G-T$ relation stay 
between the LL value 2 and the FL value 0 . Therefore, a quantitative understanding of the crossover region in such as $N(E)$ for $E$ near $T$ is necessary, before one can explain the transport experiments on the fractional edges explicitly. This will be studied in our future work.

We then turn to the discussion of the role of the Coulomb interaction at finite temperature. In a recent paper [14], we argue that the perturbative interactions between the CFs with their force-range shorter than $1 / x^{2}$ can not renormalize the exponent $g=1 / \lambda$, and the bosonization process based on the zero temperature limit of TBA is still useful. However, these arguments are not applicable to the long-range Coulomb interaction, since its phase shift function near $k=0$ is even more singular than $\Phi_{C S}(k)$, and can change the long wave length excitations of the CSM substantially. To see what happens when $T$ is not zero, let us go back to the TBA equation, where the only change is in $\Phi(k)$, which is modified by the introduction of the Coulomb interaction [27.

$$
\begin{aligned}
\Phi(k) & =\Phi_{C S}(k)+\Phi_{s i g}(k) \\
& =\Phi_{C S}(k)+C_{1} \frac{1}{k / k_{C}}+C_{2} \frac{\ln \left(k / k_{C}\right)}{k / k_{C}}
\end{aligned}
$$

where $C_{1}$ and $C_{2}$ are two constants, and $k_{C}$ is the momentum scale set by the Coulomb interaction: $k_{C}=1 / a_{B}$, with $a_{B}$ the corresponding Bohr radius.

With the inclusion of $\Phi_{\text {sig }}(k)$, one can rederive the same dispersion relation at finite $T$, if the condition $k_{T}>k_{C}$ is satisfied. The key to this invariance is the following two formulae : $\int_{-\infty}^{\infty} \Phi_{s i g}^{\prime}(k) d k=0$, and $\int_{-\infty}^{\infty} \Phi_{s i g}^{\prime}(k) k d k=0$. Therefore, one can expect a crossover from the Coulomb relevant regime to the Coulomb irrelevant regime with the increase of $T$. This helps to explain why the influence of the Coulomb interaction is not obvious in experimental observations at finite $T$, while it may change the ground state properties of CLL considerably.

In conclusion, we propose a temperature-dependent crossover picture based on the calculation of the dressed energy at finite temperature, and then apply it to the analysis of a recent tunneling experiment. We arrive at a fit to the data better than that of the LL theory. We also discuss the effects of finite temperature on the Coulomb interaction, and find a crossover behavior with the increase of temperature.

One of us(WJZ) is grateful to Hua-Gang Yan for his help in the numerical calculations. We also acknowledge the stimulating discussion with Q.Niu. This work is partly supported by the NSF of China.
[1] D.C.Tsui, H.L.Stormer and A.C.Crossard, Phys. Rev. Lett.48, 1559 (1982).

[2] M. Stone, Quantum Hall Effect( World Scientific, Singapore, 1992).

[3] B. I. Halperin, Phys. Rev. B25, 2185(1982).

[4] M. R. Geller, D. Loss, G. Kirczenow, Phys. Rev .Lett.77, 5110(1996).

[5] X. G. Wen, Phys. Rev.Lett.64, 2006(1990); Phys. Rev. B41, 12838 (1990).

[6] K. Moon, H. Yi, C. L. Kane, S. M. Girvin, and M. P. A. Fisher, Phys. Rev. Lett.71, 4381(1993).

[7] P. Fendley, A. W. W. Ludwig, and H. Saleur, Phys. Rev. lett.74, 3005(1995).

[8] F. P. Milliken, C. P. Umbach, and R. A. Webb, Solid State Commun 97, 309(1996).

[9] J. K. Jain, Phys. Rev. B 41, 7653 (1990) and references therein.

[10] G. Kirczenow, Phys. Rev. B53, 15767(1996).

[11] J. D. F. Franklin et al., Surf. Sci. 361, 17(1996).

[12] V. J. Goldman et al., Phys. Rev. B55, 4081(1997).

[13] Y. Yu, Z. Y. Zhu, Preprint cond-mat/9704124.

[14] W. J. Zheng, to be published in Int. J. Mod. Phys. B.

[15] Our work is complementary to the numerical efforts to justify CLL microscopically, see for example J. J. Palacios, A. H. Macdonald, Phys. Rev. Lett.76, 118(1996).

[16] F. Calogero, J. Math. Phys. 10, 2197 (1967).

[17] We therefore ignore the residue interactions in this letter because of their irrelevance to the critical properties of the present edge model.

[18] We stress that the crossover behavior between FL and LL is merely a finite temperature effect, while the ground state properties at $T=0$ of our edge model(CSM) are definitely of LL nature.

[19] C. N. Yang and C. P. Yang, J. Math. Phys. 10, 1115(1969).

[20] Y. S. Wu and Y. Yu, Phys. Rev. Lett. 75, 890 (1995).

[21] C. L. Kane and M. P. A. Fisher, Phys. Rev. B46, 15233(1992) ; Phys. Rev. Lett68, 12201992.

[22] A. M. Chang, L. N. Pfeiffer and K. W. West, Phys. Rev. Lett77, 2538(1996).

[23] The variation between samples should be irrelevant, because the difference between 1.75 and 1.5 is large enough to deserve intrinsic explanation.

[24] For simplicity in numerical calculations, we ignore the contributions of the crossover region.

[25] The corrections to $N(E)$ due to other factors such as the long range Coulomb interaction are possible, whose effects on the tunneling conductance remain to be verified, see U. Zulicke, A. H. Macdonald, Bull. Am. Phys. Soc. 41, 1, 79(1996).

[26] W. J. Zheng and Y. Yu, in preparation.

[27] Strictly speaking, TBA is exact only for integrable system like the CSM. The addition of Coulomb interaction here is treated perturbatively as an extension of TBA. 
FIG. 1. (a) $I-V$ characteristics for tunneling from the bulk-doped $\mathrm{n}+\mathrm{GaAs}$ into the $\nu=1 / 3$ fractional edge in a $\log$-log plot for sample 1 at $B=13.4 T$ (crosses), and samples 2 at $B=10.8 T$ (solid circles). The solid curves represent fits to the LL scaling form for $\alpha=2.75$ and 2.65, respectively[22]. (b) numerical result of $I-V \log -\log$ plot. The diamonds represent data generated by numerical integral based on the crossover picture.

FIG. 2. (a)Log-log plot of the temperature dependence of $G(T, V)$ at low voltage bias for samples 1(upper curve) and samples 2 (lower curve) at $\nu=1 / 3$. The respective voltage biases are 4.97 and $2.64 \mu \mathrm{V}$. The solid straight lines represent power laws with the respective exponents $\alpha-1$ of 1.75 and $1.5[22]$. (b)numerical result of $G(T, V)-T \log -\log$ plot based on the crossover picture, the two curves correspond to $3.3 \mu V$ (circles) and $2.1 \mu V$ (diamonds), respectively. 


\section{(a)FIG.2 of ref[19]}

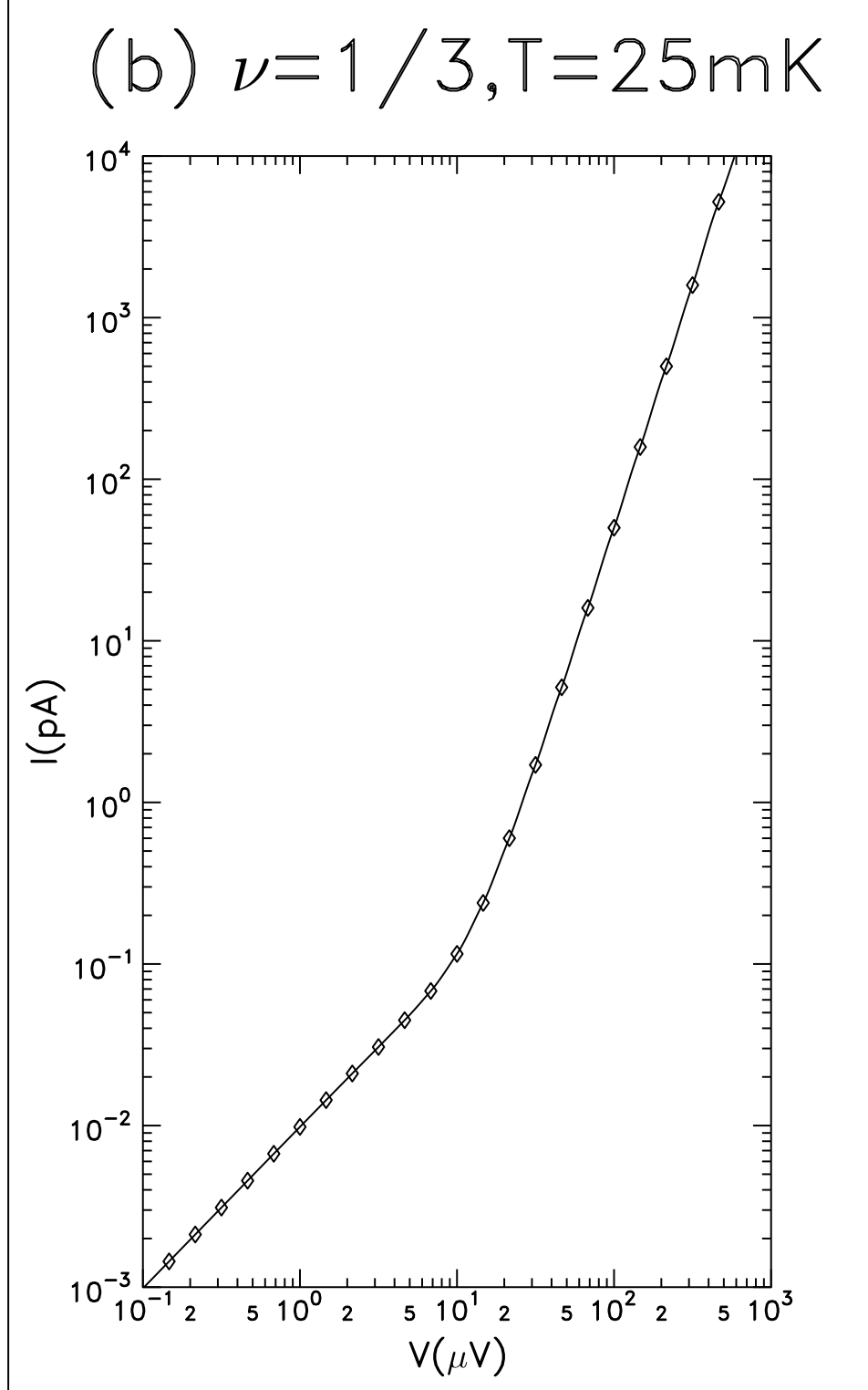




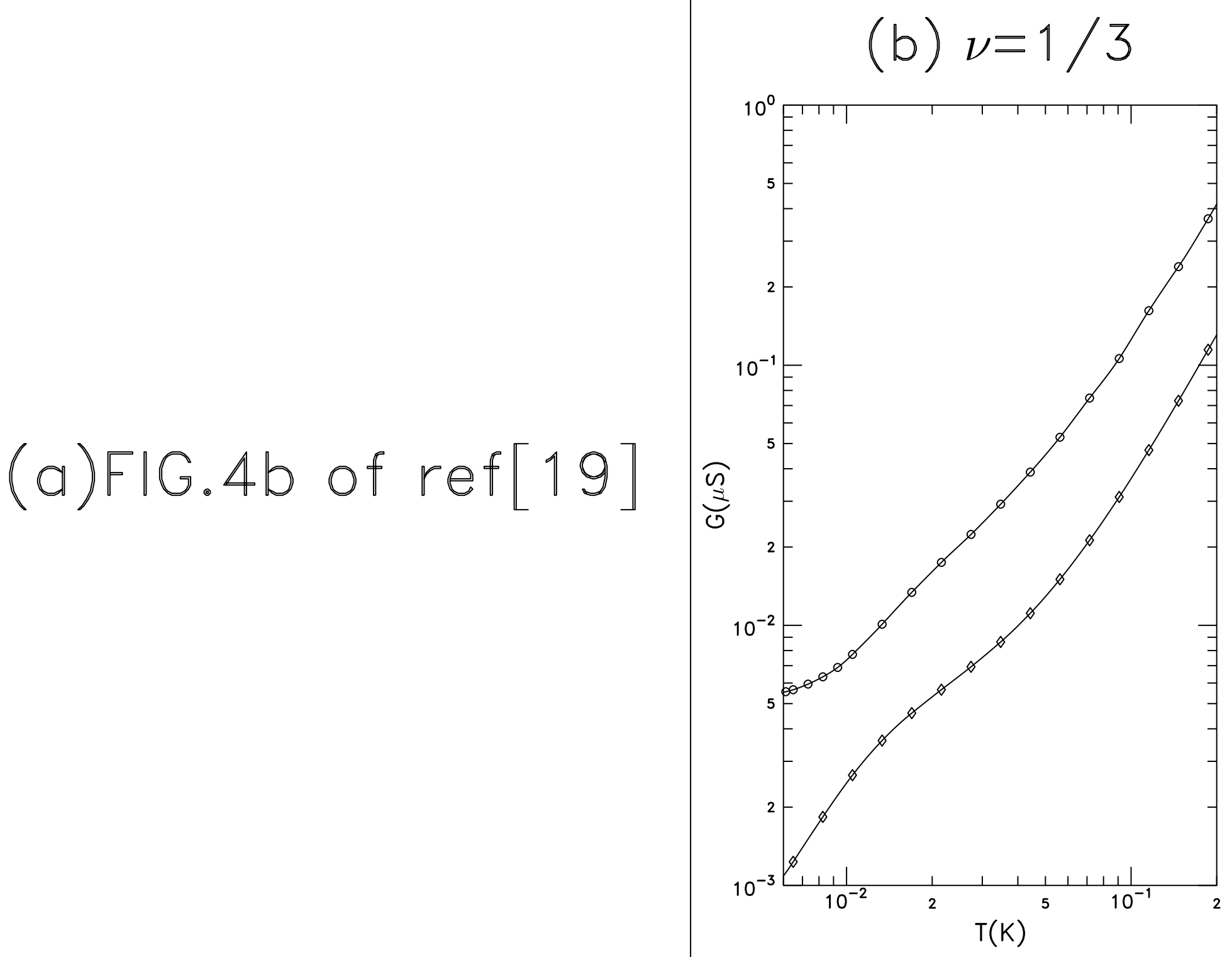

